\title{
Hybrid gold/DNA nanowire circuit with sub-10 nm nanostructure arrays
}

\author{
Jong Seob Choi ${ }^{1}$, Hye Bin Park ${ }^{2}$, Jonathan H. Tsui ${ }^{1}$, Byungyou Hong ${ }^{3}$, Deok-Ho Kim ${ }^{1,4}$ and Hyung Jin Kim²
}

\begin{abstract}
We report on a simple and efficient method for the selective positioning of Au/DNA hybrid nanocircuits using a sequential combination of electron-beam lithography (EBL), plasma ashing, and a molecular patterning process. The nanostructures produced by the EBL and ashing process could be uniformly formed over a $12.6 \mathrm{in}^{2}$ substrate with sub$10 \mathrm{~nm}$ patterning with good pattern fidelity. In addition, DNA molecules were immobilized on the selectively nanopatterned regions by alternating surface coating procedures of 3-(aminopropyl)triethoxysilane (APS) and diamond like carbon (DLC), followed by deposition of DNA molecules into a well-defined single DNA nanowire. These single DNA nanowires were used not only for fabricating Au/DNA hybrid nanowires by the conjugation of $\mathrm{Au}$ nanoparticles with DNA, but also for the formation of Au/DNA hybrid nanocircuits. These nanocircuits prepared from Au/DNA hybrid nanowires demonstrate conductivities of up to $4.3 \times 10^{5} \mathrm{~S} / \mathrm{m}$ in stable electrical performance. This selective and precise positioning method capable of controlling the size of nanostructures may find application in making sub-10 nm DNA wires and metal/DNA hybrid nanocircuits.
\end{abstract}

\section{Introduction}

Since the discovery of chemically sensitive field effect transistors with Si nanowires ${ }^{1}$, the development of metal nanoscale electronic devices has been of growing inter$\mathrm{est}^{2-5} .20$ years later, the development of techniques to fabricate well-defined 1D metal nanowires at industrial scales is still intensively investigated worldwide due to the potential utility of such wires in electronic devices that require high degrees of sensitivity ${ }^{6}$. The sensitivity of $1 \mathrm{D}$ metal nanowires for biomolecular detection has been reported as higher than that of bulk metal detection because threshold voltage and surface charge effects increase with decreasing wire width ${ }^{7}$. However, the feasibility of utilizing 1D nanowires or nanowire arrays remains in question due to difficulties associated with

\footnotetext{
Correspondence: Deok-Ho Kim (dhkim@jhu.edu) or Hyung

Jin Kim (hjkim745@geri.re.kr)

'Department of Biomedical Engineering, Johns Hopkins University, Baltimore, MD 21205, USA

2Digital Healthcare Research Center, Gumi Electronics and Information

Technology Research Institute (GERI), 350-27, Gumidaero, Gumi, Gyeongbuk 39253, South Korea

Full list of author information is available at the end of the article
}

mass production ${ }^{8}$. Moreover, precise positioning of these nanostructures is essential for enhancing their electrical sensitivity in electronic devices.

For fabricating nanowire patterns, electron-beam lithography (EBL) is one widely used top-down technology among diverse biomimetic nanopatterns technics ${ }^{6,9}$. Its high resolution, sub- $20 \mathrm{~nm}$ feature sizes, and flexible range from nanoscale to microscale makes it possible to fabricate and utilize nano-gap electrodes, molecular arrays, and nanodevices. $\mathrm{Hu}$ et al. used poly(methylmethacrylate) resists to make sub- $10 \mathrm{~nm}$ lines and wellaligned gold nanoparticle patterns on sub- $10 \mathrm{~nm}$ lines ${ }^{10}$. Sun et al. also proposed a patterning method for sub$22 \mathrm{~nm}$ Si nanowire arrays using the EBL process ${ }^{11}$. Even though nanoscale features with high reproducibility were investigated by many researchers, the EBL process still suffers from a lack of reproducibility and low throughput of sub- $10 \mathrm{~nm}$ features due to the prolonged exposure times required for large-area layouts.

Due to length controllability and binding affinity to various metal ions, alternative approaches for fabricating metal nanowires using DNA or protein assembly have 
been introduced ${ }^{12,13}$. These biomolecules can selfassemble via their charge interactions to make nanowires and can also hybridize with positively-charged metal ions or monomers. Moreover, due to its ease of experimentation and accessibility, it has emerged as a promising bottom-up approach to replace complex conventional photolithography processes. For example, Pate et al. have proposed a method of fabricating sub$10 \mathrm{~nm} \mathrm{Cu}$ nanowires by using a solution-based linear DNA template ${ }^{14}$, and Watson et al. have reported on the mechanisms behind the formation of polymer nanowires on a linear DNA template ${ }^{15}$. Although DNA-templated metal nanowires are a new bottom-up approach and have been reported as a versatile template for fabrication of well-defined metal nanostructures ${ }^{16-18}$, the technical feasibility of producing $1 \mathrm{D}$ metal/DNA nanowire arrays with precise positioning of the nanowires is still not well established $^{19,20}$.

Here, we describe a simple and efficient method to pattern sub-10 nm nanostructures with good pattern fidelity using EBL followed by a plasma ashing process. These methods enable selective positioning of sub- $10 \mathrm{~nm}$ scale structures, which when combined with a selective molecular patterning process, can be used to fabricate $\mathrm{Au} /$ DNA hybrid nanowires and nanowire arrays. As $\mathrm{Au}$ nanoparticles have been one of the most widely used nanoparticles for biosensors ${ }^{21}$, these selectivelypositioned $\mathrm{Au} / \mathrm{DNA}$ hybrid nanowire arrays can potentially serve as an effective template for the fabrication of electrical biocircuits and devices.

\section{Results and discussions}

Classical EBL technology can homogeneously make patterns with $50 \mathrm{~nm}$ feature sizes, but this method is not effective for producing sub-10 nm patterns. In this work, sub-10 nano line and lattice patterns were achieved by performing an additional ashing process following a conventional EBL process. Figure 1 provides a schematic illustration of the fabrication process and results of fabricating sub- $10 \mathrm{~nm}$ structures produced by two successive EBL and plasma ashing processes on a $\mathrm{SiO}_{2} / \mathrm{Si}$ substrate. Initial line- or lattice-patterns with $50 \mathrm{~nm}$ widths were prepared by EBL. The dimensions of these structures were proportionally decreased as plasma ashing treatment time increased (Fig. 1c-h). A final $7 \mathrm{~nm}$ width pattern could be achieved with $5 \mathrm{~min}$ of plasma ashing treatment. The ashing rate in line patterns was about $8.38 \mathrm{~nm} / \mathrm{min}$ and $7.12 \mathrm{~nm} / \mathrm{min}$ for lattice patterns (Fig. 1i, j). The small difference in ashing rates on both line- and lattice patterns is due to the turbulent flow of plasma. Plasma flow in lattice patterns could be undergoing more irregular fluctuations than in line patterns due to the more complex nanostructures, thereby resulting in a slower ashing rate.
In order to create the selective positioning of DNA molecular patterns, 3-(aminopropyl)triethoxysilane (APS) and diamond like carbon (DLC) were used as an adhesion layer and passivation layer, respectively (Fig. 2a). First, sub-10 $\mathrm{nm}$ nanostructures were prepared by consecutive EBL and plasma ashing processes as illustrated in Fig. 1. Then, a DLC layer was coated on the nanopatterned substrate by plasma enhanced chemical vapor deposition (PECVD) to form a molecular blocking layer. DLC and APS-coated layers could be confirmed by AFM topography measurements after removing the photoresist nanostructures (Fig. 2b-e). Selectively-deposited DLC layers could be observed, and these layers can be used as a passivation layer since APS molecules do not attach to DLC molecules and can only bond to nanostructures (line or lattice patterns) as initially positioned. Representative AFM images of alternating APS and DLC molecular line patterns for 4 samples prepared with different plasma ashing times show that well-aligned molecular line patterns $32 \pm 2 \mathrm{~nm}, 23 \pm 2 \mathrm{~nm}, 11 \pm 2 \mathrm{~nm}$, and $5 \pm 2 \mathrm{~nm}$ wide could be obtained after 2, 3, 4, and $5 \mathrm{~min}$ of ashing, respectively (Fig. 3 ).

APS and DLC layers play key roles in the selective attachment of DNA through strong Coulombic interactions between the $-\mathrm{NH}_{3}{ }^{+}$groups of APS and the negatively-charged phosphate backbone of DNA, and weak interactions between the hydrophobic DLC and DNA. This selective immobilization of DNA molecules along APS-coated regions can facilitate DNA nanowire assembly (Fig. 4). Prior studies had difficulty controlling the selective positioning of immobilized single DNA nanowires, and it remains a challenging issue ${ }^{20}$. However, EBL followed by plasma ashing and combined with a molecular surface patterning method can achieve precise positioning of single DNA nanowires. Prior to forming single DNA nanowire arrays on the APS coated region, we compared octadecyltrichlorosilane (OTS) to DLC in formation of single DNA nanowires as OTS is also known as a passivation coating reagent ${ }^{22,23}$. In our previous study, contact angle values for OTS-, DLC- and APS-coated surface were $95^{\circ}, 75^{\circ}$ and $55^{\circ}$, respectively ${ }^{24}$. Therefore, a DLC layer possesses hydrophobicity similar to an OTS layer. After OTS treatment of the substrate, the average RMS value of surface roughness was $0.87 \mathrm{~nm}$, which is higher than that of substrates coated with DLC $(0.13 \mathrm{~nm})$. The relatively rough surface of OTS might interrupt and confine some DNA and water molecules from moving, which leads to DNA fragments remaining on the OTS surface (Fig. 4a). On the other hand, the smoother surface of DLC appears to be more effective at preventing nonspecific attachment of DNA molecules. Most of the DNA and water molecules tended to move to the hydrophilic APS regions while the solution was evaporated or 


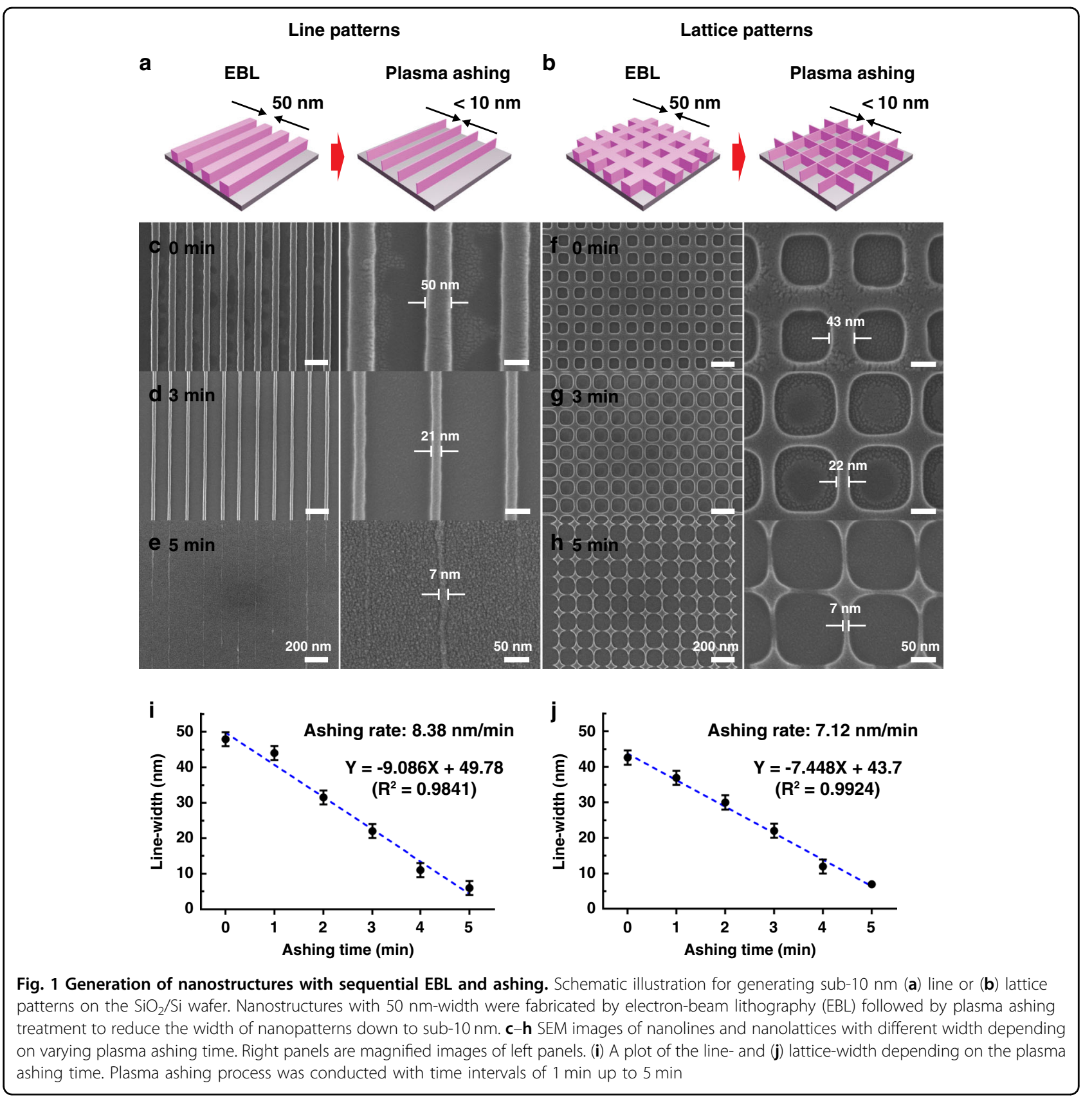

removed over time, resulting in better formation of single DNA nanowires (Fig. 4b).

To investigate the effects of the nanopattern width on DNA nanowire alignment, various widths of nanopatterns with $4 \mu \mathrm{m}, 500 \mathrm{~nm}$, and $100 \mathrm{~nm}$ were fabricated. It was found that line width after removal of the nanostructures is one of the critical parameters for achieving good DNA nanowire alignment. When the line width was relatively wide (Fig. 5a), the generated DNA nanowire formed as branched lines, which would result in branched $\mathrm{Au}$ nanowires. However, as line widths became narrower, the immobilized DNA nanowires became single lines as the reduced deposition area helped to concentrate the DNA molecules and prevent spreading. For $500 \mathrm{~nm}$-wide lines, a single DNA nanowire could be formed, albeit in a curved shape (Fig. 5b), whereas for $100 \mathrm{~nm}$-wide lines, a straight DNA nanowire along the direction of the nanopatterned line was formed (Fig. 5c).

The bonding mechanism of positively charged $\mathrm{Au}$ nanoparticles to negatively charged DNA molecules has been previously reported ${ }^{16}$. A variety of metal ions such as $\mathrm{Ag}, \mathrm{Au}, \mathrm{Pt}, \mathrm{Cu}, \mathrm{Ni}, \mathrm{Fe}, \mathrm{Co}$, and Pd react with the phosphate groups in DNA by electrostatic interaction, resulting in seed nuclei for the growth of metal nanoparticles. 


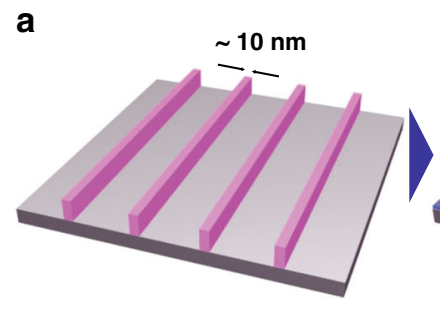

Fabrication of sub-10 nm PR patterns

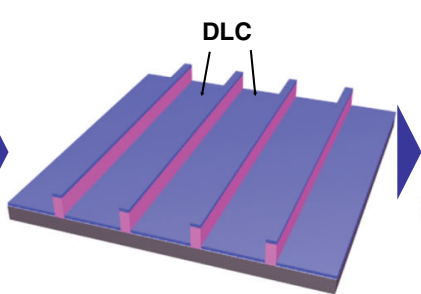

DLC coating on a substrate

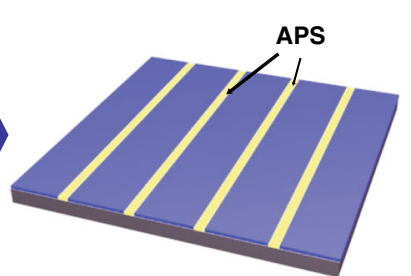

Removal of photoresist \& Selective APS coating

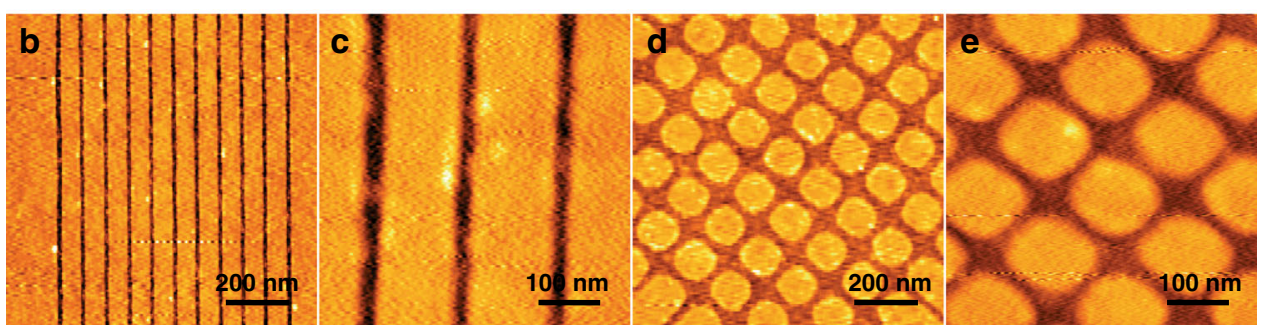

Fig. 2 Selective deposition of DLC and APS to form nanowires. a Schematic illustration for selectively aligned DLC area by using surfacepatterning techniques on the nanostructures. $\mathbf{b}$, d AFM images representing line- and lattice-patterns after DLC coating treatment. $\mathbf{c}$, e Higher magnification images of $(\mathbf{b})$ and $(\mathbf{d})$, respectively

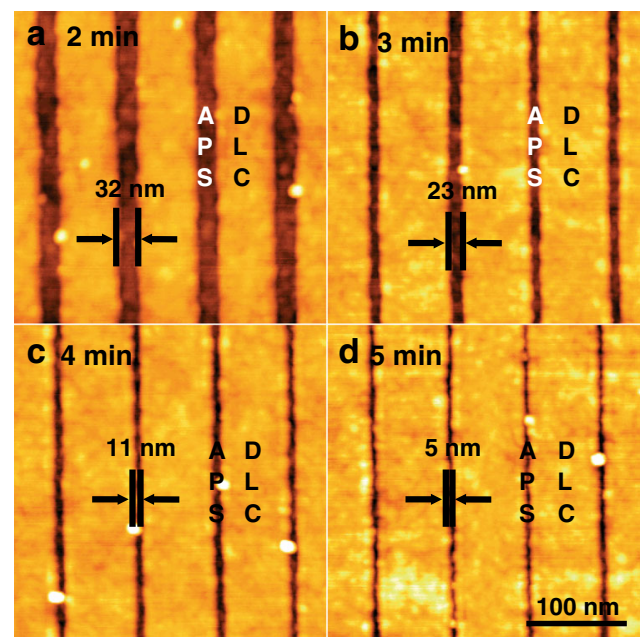

Fig. 3 Representative AFM images for line-patterns composing the alternate APS and DLC layers on a substrate after different plasma ashing times. a $2 \mathrm{~min}: 32 \pm 2 \mathrm{~nm}$, (b) $3 \mathrm{~min}: 23 \pm 2 \mathrm{~nm}$, (c) 4 min: $11 \pm 2 \mathrm{~nm}$, (d) 5 min: $5 \pm 2 \mathrm{~nm}$

However, precise positioning of a two-dimensional single metal nanowire network is still a challenging issue ${ }^{23,25}$. Here we have demonstrated the fabrication of sub-10 nm nanostructures on a 4-inch diameter wafer by using EBL and plasma ashing processes followed by alternating surface patterning methods for selectively located $\mathrm{Au} /$ DNA hybrid nanowire arrays. In total, $5 \mathrm{~nm}$ Au nanoparticles (Fig. 6a, b) were deposited onto DNA nanowires in lattice patterns (Fig. 6c, d). The average height of

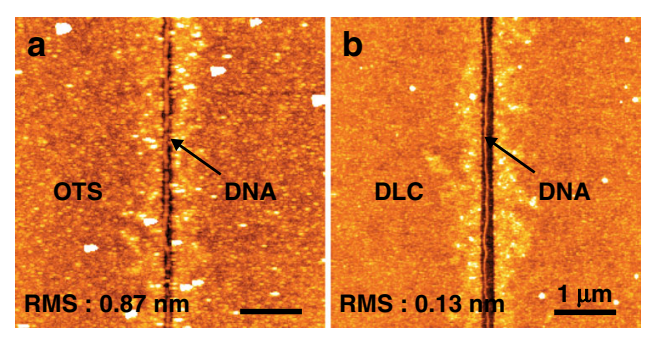

Fig. 4 A comparison of OTS and DLC as passivation layers for the formation of DNA nanowires. a Schematic illustration for selective alignment of DNA molecules on a substrate. DNA wires on (b) APS/ OTS passivation layer, and (c) APS/DLS passivation layer

produced DNA-template Au nanowires was $7 \mathrm{~nm}$ and the average width was $\sim 60 \mathrm{~nm}$ (Fig. 6e). We also attempted to reduce the width of DNA-template Au nanowires to fit sub- $10 \mathrm{~nm}$ initial nanostructures, but the $\mathrm{Au}$ nanoparticles did not properly assemble on these structures due to nanoparticle aggregation.

In most cases, this process enables the linear alignment and deposition of Au nanoparticles or clusters onto single DNA nanowires; however, if there is the presence of a nonmetallized portion or if there is no connection between the $\mathrm{Au}$ particles in the nanowire, the conductivity of the Au/DNA hybrid nanowire or network would be negatively impacted. To measure the degree of conductivity of Au/DNA nanowires and to check their integrity, a Kelvin probe force microscope (KFM) study was carried out to obtain surface potential values. Like 
a $4 \mu \mathrm{m}$
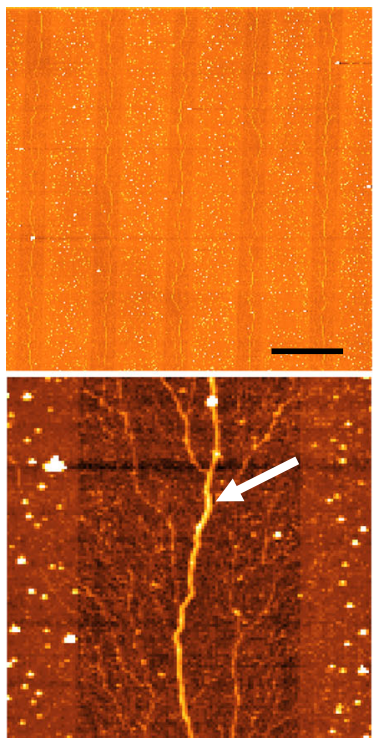

b $500 \mathrm{~nm}$
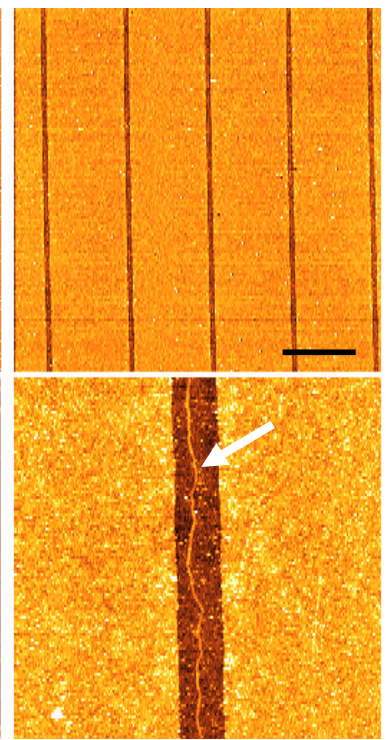

C $100 \mathrm{~nm}$

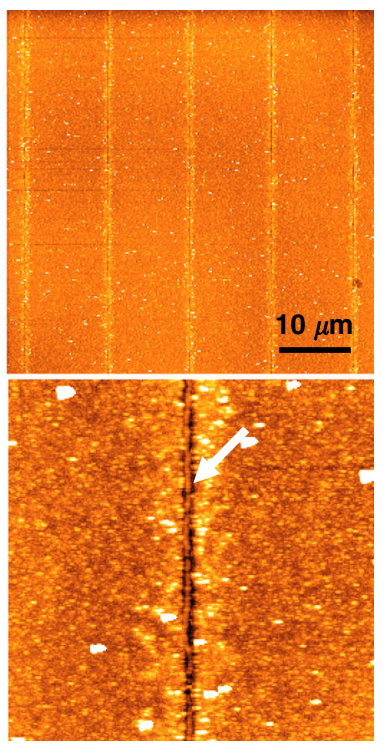

Fig. 5 Nanopattern line width influences the alignment of DNA nanowires. (top) DNA alignment on the APS regions. Bottom images are magnified from top images. White arrows indicate the DNA nanowire on the APS region. a $4 \mu \mathrm{m}$, (b) $500 \mathrm{~nm}$, (c) $100 \mathrm{~nm}$. Bottom image in (c) is the same with Fig. 4 (b)
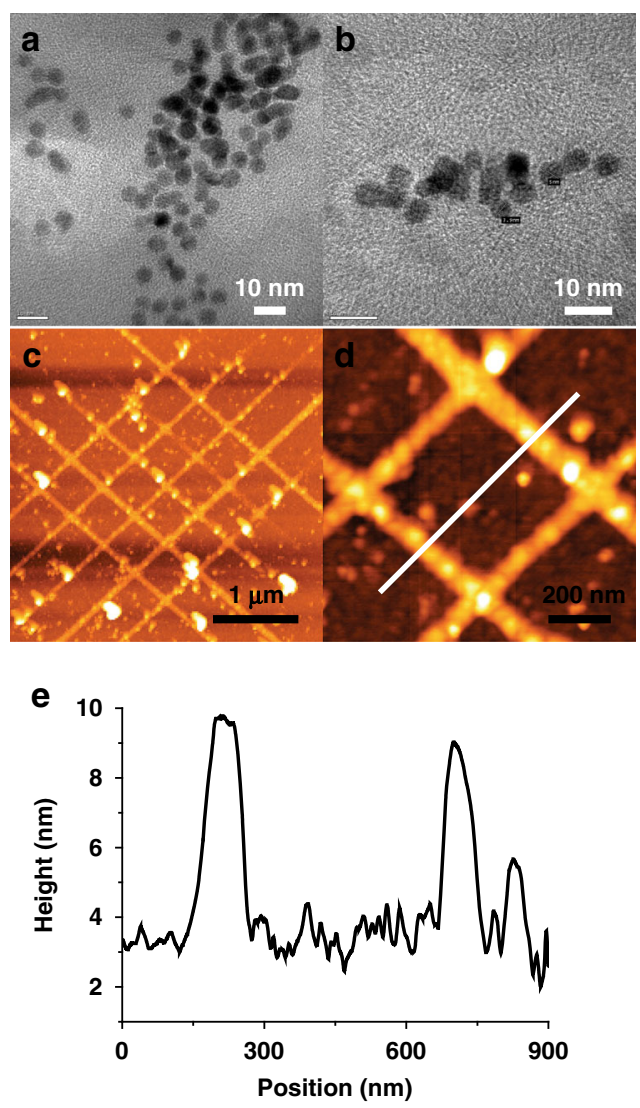

Fig. 6 Fabrication of DNA-Au hybrid nanowires. $\mathbf{a}$, $\mathbf{b}$ Representative TEM images of Au nanoparticles used for assembly of Au nanowires. $\mathbf{c}$, d AFM topography for DNA-Au hybrid nanowires aligned on the $\mathrm{SiO}_{2} / \mathrm{Si}$ substrate. e A height profile represented by the white line in (d) with the topographical measurements obtained with AFM, KFM analysis also showed that the crosshatched areas of the $\mathrm{Au}$ nanowire lattices were better assembled and appeared more distinctly than the line patterns (Fig. $7 \mathrm{a}, \mathrm{d})$. It is likely that this is due to a higher positive charge density at the crosshatch area compared to that along individual lines, resulting in a large amount of negativelycharged DNA molecules being immobilized on the crosshatched sections. As a result, most of the positivelycharged $\mathrm{Au}$ nanoparticles were then preferentially localized to the greater number of DNA molecules in these areas. Measured maximum surface potential was $\sim 6 \mathrm{mV}$ along the $\mathrm{Au} / \mathrm{DNA}$ nanowire. The measurement of surface potential of the nanowire-based sensors is a crucial parameter for understanding and predicting the performance of nanowire sensors ${ }^{26}$, as reacting metal nanowires with biomolecules via charge interactions can result in a subsequent alteration of nanowire conductivity.

To further assess the electrical properties of fabricated hybrid Au/DNA nanowires, two Pt electrodes were fabricated at the ends of nanowire arrays (Fig. 8a). The linear relationship between current and voltage shows an ohmic behavior, an indication that Au/DNA hybrid nanowires possess metallic properties (Fig. 8b). The conductivity of $\mathrm{Au} / \mathrm{DNA}$ hybrid nanowires was about $4.3 \times 10^{5} \mathrm{~S} / \mathrm{m}$, which is 2 orders of magnitude lower than pure $\mathrm{Au}$ electrodes ${ }^{27}$. In addition, there was no difference in conductivity between the two sets of nanowire arrays $(1,2)$ shown in Fig. 8a. This is likely due to there being no nonmetallized portions or connection breaks between $\mathrm{Au}$ nanoparticles in the nanowires. The reported conductivity 

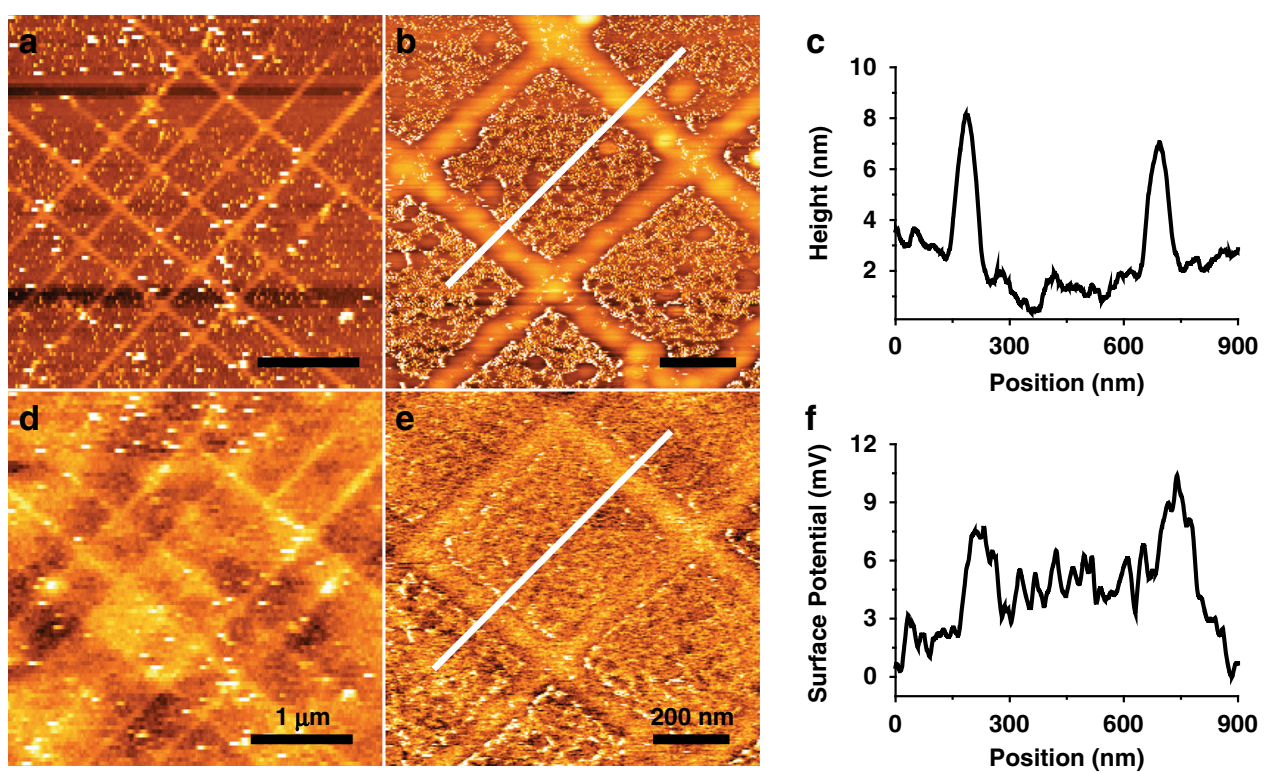

Fig. 7 KFM study for high resolution surface potential and topography mapping. $\mathbf{a}$, b KFM topography mapping of hybrid Au/DNA Nanowire array. c A height profile of the white line in (b). d, e KFM surface potential images. $\mathbf{f}$ A surface potential profile represented by the white line in (e)
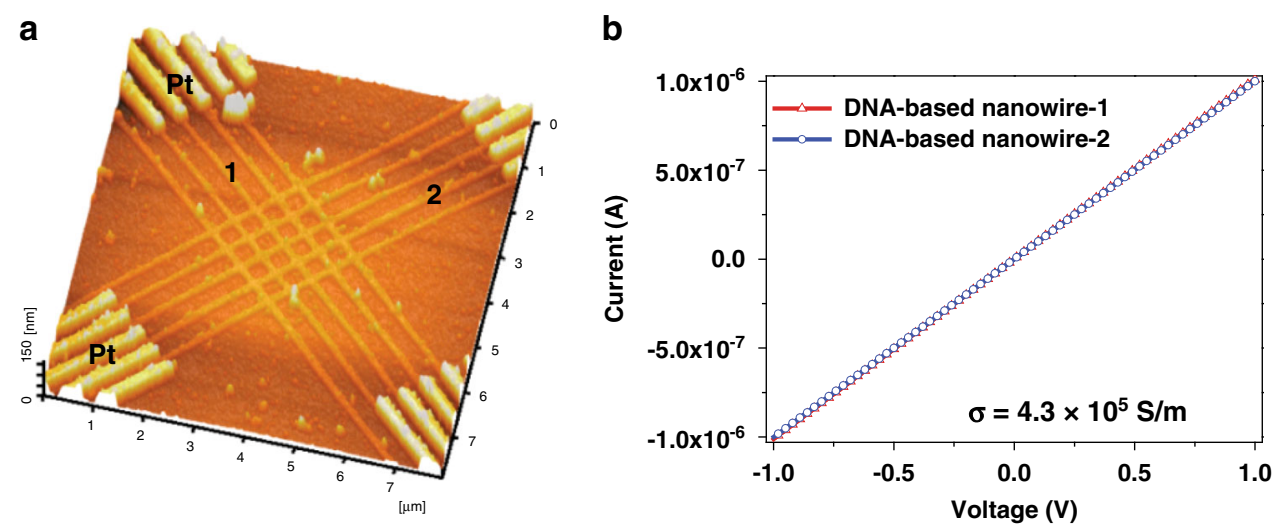

Fig. 8 Nanocircuit fabrication using Au/DNA hybrid nanowire arrays fixed between two Pt electrodes. a AFM topography image with the numbers 1 and 2 indicating each nanowire line array. $\mathbf{b}$ Electrical properties (current-voltage) of nanocircuits made from Au/DNA hybrid nanowires

of linear $\mathrm{Au} / \mathrm{DNA}$ nanowires fabricated using a similar method $(3.2 \times 104 \mathrm{~S} / \mathrm{m})$ was lower than that of the grid patterns of hybrid nanowires fabricated in this study ${ }^{28}$. This is likely due to the high concentration of DNA molecules at the grid intersections that would result in a greater density of Au nanoparticles that are present than there would be in a linear Au/DNA nanowire. Moreover, electrode sensitivity is greatly related to the device quality such as electrical conductivity and impedance ${ }^{29}$. Surface area and charge potentials at the surface are one of the important parameters to enhance the electrode performance in recording sensitivity. Surface area of grid patterns is higher than that of line patterns. We believe that this is a result of a relatively high accumulation of potentials at the surface areas of the grid Au/DNA patterns that can then cause a high density of $\mathrm{Au}$ nanoparticles to be attached to the biomolecules.

Overall, uniformly formed sub-10 nm structures over a 4-inch wafer-size with good pattern fidelity was achieved by using a combination of EBL and plasma ashing. By an additional surface patterning process, selectively-aligned and positioned hybrid Au/DNA nanowires and arrays could be fabricated onto sub$10 \mathrm{~nm}$ structures. Our approach to fabricating Au/DNA nanocircuits is a promising technique for nanoelectronics applications that require precise assembly and positioning of electrodes and wiring. These Au/DNA hybrid nanocircuits could be further developed and 
applied as biomedical and environmental sensors through integration with appropriate technologies such as nano/microfluidics and molecular biology for earlydetection diagnostics ${ }^{17,30}$.

\section{Methods}

Aligned hybrid DNA/Au nanowires were produced as follows: (1) A ma-N2401 negative tone electron beam resist (MicroResist Technology) was coated on top of a silicon substrate with a $200 \mathrm{~nm}$-thick oxide layer (WaferWorks Corp.). The thickness of the resist was held at $\sim 100 \mathrm{~nm}$ by optimizing the spin-coating speed (1000 rpm for $120 \mathrm{~s}$ ). In order to form nanopatterns with uniform intervals, the resist was exposed to electron beam light (JEOL, JBX6000FS) with a dose of $200 \mu \mathrm{C} / \mathrm{cm}^{2}$ at $25 \mathrm{kV}$. The exposed layer was developed with AZ726 (MicroChemicals) for $80 \mathrm{~s}$ and then rinsed with double distilled water for $60 \mathrm{~s}$. As a result, $50 \mathrm{~nm}$ width nanopatterns were produced with intervals of $100 \mathrm{~nm}$. (2) The nanopatterned structures were ashed by using a chemical dry etcher (Tokuda Co., CDE-7-3) with $\mathrm{O}_{2}(1500 \mathrm{sccm})$, $\mathrm{N}_{2}(500 \mathrm{sccm})$, and $\mathrm{CF}_{4}(100 \mathrm{sccm})$ at $50 \mathrm{~W}$ microwave power $(2.45 \mathrm{GHz})$. (3) Then, a DLC thin film with a thickness of $5 \mathrm{~nm}$ was deposited on the nano-patterned substrate by RF PECVD with methane $\left(\mathrm{CH}_{4}\right)$ and hydrogen $\left(\mathrm{H}_{2}\right)$ gas at $150 \mathrm{~W}$ RF power $(13.56 \mathrm{MHz})$. (4) After lift-off of the nanopatterned structures, the substrate was immersed in a $0.1 \mathrm{mM}$ 3-aminopropyltriethoxysilane (Sigma-Aldrich) solution diluted in anhydrous toluene (Sigma-Aldrich) for $30 \mathrm{~min}$. (5) A $\lambda$-DNA (Bio Basic Inc., $48,502 \mathrm{bps}$ ) solution of $30 \mathrm{~mL}$ was prepared in a concentration of $10 \mathrm{ng} / \mathrm{mL}$ by TE buffer $(10 \mathrm{mM}$ tris- $\mathrm{HCl}$ and $1 \mathrm{mM}$ EDTA, pH 8.0; Noble Biosciences. Inc.) and deposited on APS regions and allowed to react for $3 \mathrm{~min}$. (6) DNA molecules were treated with an aniline (SigmaAldrich)-capped gold nanoparticle (AN-AuNP) solution of $60 \mu \mathrm{L}$ to form hybrid nanowires and, after $30 \mathrm{~min}$, the samples were rinsed with double distilled water. The samples were observed with an atomic force microscope (AFM, SII Nanotechnology, SPA 400) and field emission-scanning electron microscope (FE-SEM, JSM-7610F).

In order to measure electrical properties of $\mathrm{Au} / \mathrm{DNA}$ nanowire arrays, titanium/platinum (Ti/Pt) electrodes ( $250 \mathrm{~nm}$ in width, $250 \mathrm{~nm}$ in space between Ti/Pt electrodes) on each end of Au/DNA chain was fabricated by conventional photolithography. Then, thermal evaporation was conducted to sequentially deposit $50 \mathrm{~nm}$ of $\mathrm{Ti}$ and $150 \mathrm{~nm}$ of Pt on the entire wafer. After removing the photoresist underneath $\mathrm{Ti} / \mathrm{Pt}$ layer, $\mathrm{Ti} / \mathrm{Pt}$ electrodes were selectively positioned and connected with $\mathrm{Au} / \mathrm{DNA}$ nanowires. The conducting property was characterized using a precise semiconductor parameter analyzer (SnMICVMS100).

\section{Acknowledgements}

This work was supported by the Ministry of Health \& Welfare, Republic of Korea (HI19C0642), by the Ministry of Trade, Industry and Energy (MOTIE) and Korea Institute for Advancement of Technology (KIAT) through the International Cooperative R\&D program (Project No. P0004638), and by the Gumi Core Components and Materials Technology Development Program of the Gumi Regional Government (Core Project-563 grant, 2019).

\section{Author details}

'Department of Biomedical Engineering, Johns Hopkins University, Baltimore, MD 21205, USA. ${ }^{2}$ Digital Healthcare Research Center, Gumi Electronics and Information Technology Research Institute (GERI), 350-27, Gumidaero, Gumi, Gyeongbuk 39253, South Korea. ${ }^{3}$ College of Information and Communication Engineering, Sungkyunkwan University, Suwon 440-746, South Korea.

${ }^{4}$ Department of Medicine, Johns Hopkins University School of Medicine, Baltimore, MD 21205, USA

\section{Author contributions}

J.S.C. nanofabricated the array and wrote the manuscript. H.B.P. analyzed the AFM and KFM images, J.H.T. assisted with data analysis and manuscript writing, B.H. developed the overall concept of the work, D.-H.K. wrote and discussed the manuscript, and H.J.K. performed all experiments, wrote and discussed the manuscript, and supervised this project.

\section{Conflict of interest}

The authors declare the following competing financial interest(s): D.-H.K. is a scientific founder and equity holder of Curi Bio.

Received: 30 November 2019 Revised: 30 June 2020 Accepted: 6 August 2020

Published online: 02 November 2020

\section{References}

1. Cui, Y. Nanowire nanosensors for highly sensitive and selective detection of biological and chemical species. Science 293, 1289-1292 (2001).

2. Li, Y., Qian, F., Xiang, J. \& Lieber, C. M. Nanowire electronic and optoelectronic devices. Mater. Today 9, 18-27 (2006).

3. Colinge, J.P. et al. Nanowire transistors without junctions. Nat. Nanotechnol. 5, 225-229 (2010).

4. Tapio, K. et al. Toward single electron nanoelectronics using self-assembled DNA structure. Nano Lett. 16, 6780-6786 (2016).

5. Oener, S. Z. et al. Metal-insulator-semiconductor nanowire network solar cells. Nano Lett. 16, 3689-3695 (2016).

6. Hobbs, R. G., Petkov, N. \& Holmes, J. D. Semiconductor nanowire $f$ abrication by bottom-up and top-down paradigms. Chem. Mater. 24, 1975-1991 (2012).

7. Elfström, $\mathrm{N}$. et al. Surface charge sensitivity of silicon nanowires: size dependence. Nano Lett. 7, 2608-2612 (2007).

8. Park, H.-Y. et al. M-DNA/transition metal dichalcogenide hybrid structurebased Bio-FET sensor with ultra-high sensitivity. Sci. Rep. 6, 35733 (2016).

9. Kim, D.-H., Lee, H., Lee, Y. K., Nam, J.M. \& Levchenko, A. Biomimetic nanopatterns as enabling tools for analysis and control of live cells. Adv. Mater. 22, 4551-4566 (2010).

10. Hu, W. (Walter)., Sarveswaran, K., Lieberman, M. \& Bernstein, G.H. Sub-10 $\mathrm{nm}$ electron beam lithography using cold development of poly(methylmethacrylate). J. Vac. Sci. Technol. B Microelectron. Nanom. Struct. 22, 1711 (2004).

11. Sun, M.C. et al. Patterning of Si nanowire array with electron beam lithography for sub-22nm Si nanoelectronics technology. Microelectron. Eng. 110, 141-146 (2013).

12. Yan, H. DNA-templated self-assembly of protein arrays and highly conductive nanowires. Sci. (80-). 301, 1882-1884 (2003).

13. Mou, Y., Yu, J.-Y., Wannier, T. M., Guo, C.-L. \& Mayo, S. L. Computational design of co-assembling protein-DNA nanowires. Nature 525, 230-233 (2015)

14. Pate, J. et al. Solution-based DNA-templating of sub- $10 \mathrm{~nm}$ conductive copper nanowires. J. Mater. Chem. C. 2, 9265-9273 (2014). 
15. Watson, S. M. D., Galindo, M. A., Horrocks, B. R. \& Houlton, A. Mechanism of formation of supramolecular DNA-templated polymer nanowires. J. Am. Chem Soc. 136, 6649-6655 (2014)

16. Watson, S. M. D., Pike, A. R., Pate, J., Houlton, A. \& Horrocks, B. R. DNAtemplated nanowires: morphology and electrical conductivity. Nanoscale 6, 4027-4037 (2014).

17. Kim, J. et al. Applications, techniques, and microfluidic interfacing for nanoscale biosensing. Microfluid. Nanofluidics 7, 149-167 (2009).

18. Kuzyk, A. et al. Reconfigurable 3D plasmonic metamolecules. Nat. Mater. 13, 862-866 (2014).

19. Russell, C. et al. Gold nanowire based electrical DNA detection using rolling circle amplification. ACS Nano 8, 1147-1153 (2014).

20. Eidelshtein, $\mathrm{G}$. et al. Synthesis and properties of novel silver-containing DNA molecules. Adv. Mater. 28, 4839-4844 (2016).

21. Cao, J., Sun, T. \& Grattan, K. T. V. Gold nanorod-based localized surface plasmon resonance biosensors: a review. Sens. Actuators B Chem. 195, 332-351 (2014).

22. Kim, $\mathrm{H}$. et al. Synthesis and characteristics of $\mathrm{NH} 2$-functionalized polymer films to align and immobilize DNA molecules. Nanoscale Res. Lett. 7, 30 (2012).

23. Zhang, Y. et al. Single gold nanowire electrodes and single Pt@Au nanowire electrodes: electrochemistry and applications. Chem. Commun. 53, 2850-2853 (2017).
24. Kim, H. J., Yun, D. Y., Choi, W. S. \& Hong, B. Selective assembly of DNA using DLC film as passivation layer for the application to nano-device. Diam. Relat. Mater. 18, 1015-1018 (2009).

25. Yang, J., Bao, C., Zhu, K., Yu, T. \& Xu, Q. High-performance transparent conducting metal network electrodes for perovksite photodetectors. ACS Appl. Mater. Interfaces 10, 1996-2003 (2018).

26. Ambhorkar, P. et al. Nanowire-based biosensors: from growth to applications. Micromachines 9, 679 (2018).

27. Naghdi, S., Rhee, K., Hui, D. \& Park, S. A review of conductive metal nanomaterials as conductive, transparent, and flexible coatings, thin films, and conductive fillers: different deposition methods and applications. Coatings $\mathbf{8}$, 278 (2018).

28. Kim, H. J., Roh, Y. \& Hong, B. Selective formation of a latticed nanostructure with the precise alignment of DNA-templated gold nanowires. Langmuir $\mathbf{2 6}$, 18315-18319 (2010)

29. Rajan, N. K., Routenberg, D. A. \& Reed, M. A. Optimal signal-to-noise ratio for silicon nanowire biochemical sensors. Appl. Phys. Lett. 98, 264107 (2011).

30. Liu, F., Piao, Y., Choi, J. S. \& Seo, T. S. Three-dimensional graphene micropillar based electrochemical sensor for phenol detection. Biosens. Bioelectron. 50, 387-392 (2013) 